\title{
A Multiscale Intracranial Pressure Signal Simulator
}

\author{
Federico Wadehn ${ }^{1}$, David J Mack ${ }^{2}$, Emanuela Keller ${ }^{2}$, Thomas Heldt ${ }^{3}$ \\ ${ }^{1}$ Swiss Federal Institute of Technology Zurich (ETH), Switzerland \\ ${ }^{2}$ University Hospital Zurich, Switzerland \\ ${ }^{3}$ Massachusetts Institute of Technology, Cambridge, MA, USA
}

\begin{abstract}
Intracranial pressure (ICP) is a key vital sign whose temporal behavior across multiple time scales is increasingly being studied, e.g., to assess brain compliance or to estimate cerebral perfusion. In this paper we present a multiscale ICP signal simulator that acts on three time scales: Long-term ICP trends (minutes to hours), respiration-induced changes in ICP and single ICP pulses. The long-term trends are based on a mechanistic ICP model, whereas the respiration-induced and intrabeat changes are represented through phenomenological models. The latter capture waveform properties extracted from real patients in our ICP database as well as waveform features described in the literature. Potential use cases of the simulator include the evaluation of ICP signal analysis algorithms and testing of patient-monitor alarm settings.
\end{abstract}

\section{Introduction}

Intracranial pressure (ICP) is a key physiological vital sign and is monitored in patients with severe neurological disorders, such as subarachnoid haemorrhage or traumatic brain injury [1]. High resolution ICP recordings are increasingly being used to assess brain compliance [2], to benchmark non-invasive ICP estimation algorithms $[3,4]$ and for assessing cerebral perfusion [5].

Due to the invasiveness of the measurement procedure, ICP waveform data are not as readily available as photoplethysmographic or electrocardiographic (ECG) waveform data. Especially for the latter, there exist a large variety of simulators. Examples from the literature include the dynamical ECG model [6], and commercial solutions include the Fluke ProSim patient simulator series (Fluke Biomedical, WA, USA). Simulators with a comparable level of physiological realism, however, are not available for ICP. This makes an objective evaluation of ICP analysis algorithms difficult. In addition to providing a ground truth for benchmarking algorithms, such simulators have the potential to be employed for testing and calibrating patient monitors in critical care facilities.
Existing ICP models and simulators fall into two categories: physiological models [7] and phenomenological simulators [8]. The ICP and arterial blood pressure (ABP) models presented in [8] mimic real waveforms by capturing statistical features of ICP and ABP signals. This is achieved by modeling the pulsatile component of these signals with a two-component Fourier series (base frequency and first harmonic), whose amplitude is modulated by a sinusoid at the respiratory frequency. On the other side of the spectrum, there are detailed physiological ICP models such as [7] or those summarized in [9], that describe the interaction of cerebral blood, cerebrospinal fluid and the various control loops which try to keep blood flow matched to the metabolic demand. These models mostly describe slow ICP dynamics on the order of minutes.

In this work, we combine these two approaches to obtain an ICP simulator ${ }^{1}$, that represents ICP waveform features across three time scales: slow ICP dynamics, respirationinduced changes in ICP and single ICP pulses of varying morphology. Finally, models for common types of cardiac arrhythmias as well as artifacts such as catheter flushes and signal drop-outs have been included to increase the degree of realism of the simulated waveforms.

\section{The Multiscale ICP Simulator}

The simulator's slow temporal dynamics (minutes to hours) are based on a physiological ICP model [7]. To this, we add respiration-induced periodic changes in ICP, modeled by a sinusoid whose amplitude is monotonically increasing with mean ICP. Single ICP pulses are modeled with a three-component Gamma mixture model, whose location and scale parameters also depend on mean ICP.

\subsection{Slow ICP Dynamics}

Cerebral blood flow (CBF) is determined by ABP, ICP, venous pressure $(\mathrm{Pv})$ as well as vascular resistance and

${ }^{1}$ MATLAB code is available at https://github.com/ Effesian/Multiscale-Intracranial-Pressure-Simulator. 


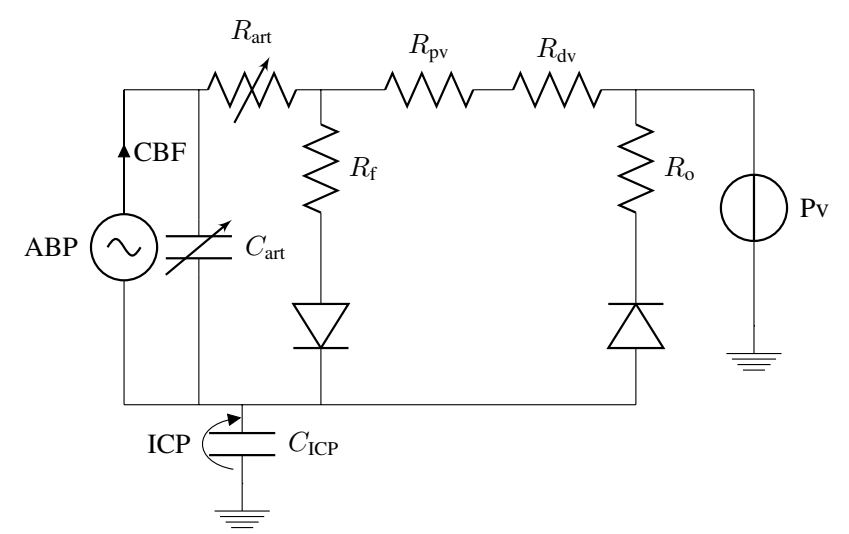

Figure 1: Electric circuit analogy of the Ursino-Lodi ICP model with variable arterial resistance $R_{\text {art }}$ and compliance $C_{\text {art }}$, controlled by cerebral autoregulation. The venous side is modeled respectively by the proximal and distal resistance $R_{\mathrm{pv}}$ and $R_{\mathrm{dv}}$ and the venous pressure Pv. CSF is produced by filtering capillary blood through $R_{f}$ and then reabsorbed into the venous side through $R_{o}$. Intracranial compliance and pressure are denoted as $C_{\mathrm{ICP}}$ and ICP.

compliance. Various control mechanisms, known as cerebral autoregulation, are in place to keep CBF matched to cerebral metabolic demand. The Ursino-Lodi model (Fig. 1 ) is an electric circuit analogy for CBF and cerebrospinal fluid (CSF) flow [7]. It represents a feedback control mechanism adapting arterial resistance and compliance to keep CBF at a desired set point. Depending on the choice of parameters, the model can display self-sustained ICP oscillations that mimic transient episodes of intracranial hypertension known as plateau waves (Figs. 3-5). These are episodes of intracranial hypertension triggered by a vasodilatory cascade [7]. The slow ICP dynamics, termed $\mathrm{ICP}_{\text {trend }}[\cdot]$ in our simulator, are obtained by solving the differential equations implied by the circuit model in Fig. 1, using the nominal parameters from [7]. For this, we use the Runge-Kutta (RK4) routine with a step size of 1s (MATLAB R2017b, The MathWorks, MA, USA).

\subsection{Respiration-induced changes in ICP}

Respiration affects various cardiovascular parameters. Changes in intrathoracic pressure influence cardiac filling, which in turn influences cardiac output and thus ABP [10]. ICP is modulated by respiration too (Figs. 2 and 6); on the arterial side by changes in ABP and on the venous side, by changes in venous pressure, which determine outflow from cerebral veins.

Similarly to [8], for modeling the influence of respiration on ICP, we do not resort to a mechanistic (physiological) model, but instead create a waveform with a similar shape to real recordings. We model respiration-induced ICP modulations with a sinusoid whose frequency is the respiratory rate $f_{\text {resp }}=1 / T_{\text {resp }}$. $\mathrm{ICP}_{\text {resp }}$ during the $\ell$-th res- piration cycle covers the time window

$$
w_{\ell, \ell+1}^{\text {resp }}=\left[\ell T_{\text {resp }}:(\ell+1) T_{\text {resp }}\right]
$$

and has an amplitude that increases with mean ICP according to

$$
A_{\text {resp }}[\ell]=k_{\text {resp }} \sqrt{\left|\operatorname{mean}\left(\mathrm{ICP}_{\text {trend }}\left[w_{\ell, \ell+1}^{\text {resp }}\right]\right)\right|},
$$

with $k_{\text {resp }}$ being a proportionality constant. Note that for very small or negative ICP, (2) is not a good model for ICP variations anymore. Finally we get

$$
\mathrm{ICP}_{\text {resp }}[t]=A_{\text {resp }}[\ell] \cdot \sin \left[2 \pi f_{\text {resp }} \cdot t\right], t \in w_{\ell, \ell+1}^{\text {resp }} .
$$

\subsection{ICP Pulse Amplitude and Shape}

Both ICP pulse amplitude and waveform morphology are known to change with mean ICP, in our case with $\overline{\mathrm{ICP}}[t]=\mathrm{ICP}_{\text {trend }}[t]+\mathrm{ICP}_{\text {resp }}[t]$. The ICP pulse commonly consist of three peaks (Figs. 2, 6 and 8). These peaks are called P1 (percussion wave originating from systolic ABP pulsations), P2 (reflected CSF pressure wave) and P3 (due to venous pulsations) [11]. High ICP and reduced brain compliance are thought to lead to an elevation of the P2 amplitude over the P1 amplitude [11] (Fig. 7). According to the exponential craniospinal pressure-volume relation [12], the ICP pulse amplitude increases with mean ICP. To model the pulsatile component $\mathrm{ICP}_{\text {pulse }}$, we generate single ICP pulses, that are subsequently concatenated. Single pulses are modeled by a three-component Gamma mixture model with the $\ell$-th pulse being

$$
\widetilde{\operatorname{ICP}}_{\text {pulse }}[t, \ell]=A_{\text {pulse }}[\ell] \sum_{i=1}^{3} w_{i} \Gamma\left(t-\tau_{i} ; \alpha_{i}, \beta_{i}\right)
$$

with $t \in[0, \infty]$ and where $\Gamma(t-\tau ; \alpha, \beta)$ is a Gamma distribution with shape parameter $\alpha$, rate parameter $\beta$ and $\tau$, accounting for the shift between peaks P1, P2 and P3. Each pulse is cropped to a finite duration, scaled to the length of the interbeat-interval $T_{\mathrm{IBI}}$ determined by the heart rate, and (4) is detrended to arrive at a zero-mean $\mathrm{ICP}_{\text {pulse }}$ in the final addition step of (7). The pulse amplitude $A_{\text {pulse }}[\ell]$ is a function of mean ICP during the $\ell$-th beat

$$
A_{\text {pulse }}[\ell]=k_{1}^{\text {pulse }} \exp \left(\operatorname{mean}\left(\overline{\mathrm{ICP}}\left[\ell T_{\mathrm{IBI}}:(\ell+1) T_{\mathrm{IBI}}\right]\right) \cdot k_{2}^{\text {pulse }}\right)
$$

where the parameters $k_{1}^{\text {pulse }}$ and $k_{2}^{\text {pulse }}$ can be fit to available ICP recordings. The relative weights $w_{i}$ are used to adapt the shape of ICP pulses. To model changes in P2 and P3 as a function of mean ICP we use

$$
w_{i}=\gamma_{i} \cdot \operatorname{mean}\left(\overline{\mathrm{ICP}}\left[\ell T_{\mathrm{IBI}}:(\ell+1) T_{\mathrm{IBI}}\right]-10\right)+w_{i}^{0},
$$

where $i \in\{2,3\}$ and $w_{i}^{0}$ are the weights of $\mathrm{P} 2$ and $\mathrm{P} 3$, respectively, at ICP $=10 \mathrm{mmHg}$ and $\gamma_{i}$ is a constant. Concatenating the ICP pulses results in the signal $\mathrm{ICP}_{\text {pulse }}$. Note that, as for the respiration-induced ICP changes, (5) works only well for non-negative ICP. 


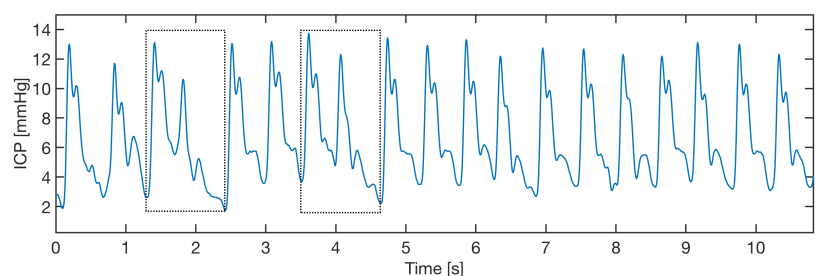

Figure 2: Real ICP recording in a mechanically ventilated patient with respiration-induced variations (e.g., from $t=4.5 \mathrm{~s}$ to $t=7 \mathrm{~s}$ ) and ventricular premature beats (dotted boxes) triggered every 10th beat.

\subsection{Composite ICP Model}

The ICP signal is finally obtained by adding the three components:

$$
\mathrm{ICP}[t]=\mathrm{ICP}_{\text {trend }}[t]+\mathrm{ICP}_{\text {resp }}[t]+\mathrm{ICP}_{\text {pulse }}[t] .
$$

Note that since both $\mathrm{ICP}_{\text {resp }}$ and $\mathrm{ICP}_{\text {pulse }}$ have parameters that depend on $\mathrm{ICP}_{\text {trend }}$, the model is not additive, but contains non-linear dependencies. Finally, to avoid sharp edges in the signal where the ICP pulses are concatenated, we apply a 10-point moving average filter to the signal.

For our simulations we used: $f_{\text {resp }}=10$ breaths $/ \mathrm{min}$ in (3) and $k_{\text {resp }}=0.2 \sqrt{\mathrm{mmHg}}$ in (2). The shift parameters for ICP pulses in (4) are: $\tau_{1}=0, \tau_{2}=1, \tau_{3}=2$ and the shape parameters $\alpha_{1}=5, \alpha_{2}=6, \alpha_{3}=8, \beta_{1}=0.2$, $\beta_{2}=0.2$ and $\beta_{3}=0.3$. In (5), we use $k_{1}^{\text {pulse }}=10 \mathrm{mmHg}$ and $k_{2}^{\text {pulse }}=80$ and in (6) $w_{2}^{0}=1.2, w_{3}^{0}=1.1, \gamma_{2}=0.01$ and $\gamma_{3}=0.009$.

\subsection{Arrhythmia and Artifact Models}

ICU patients often present with cardiac arrhythmias and to represent some ectopic activity, we added triggers of premature ventricular beats to the simulator (Fig. 9). The premature beats start about $25 \%$ earlier than regular beats, have a 1.5 times longer duration and result in a $50 \%$ reduced pulse amplitude.

Also, common artifacts in ICP monitoring include: signal drops due to sensor malfunction (Fig. 9) and catheter flushes (Fig. 10), for which we used the hyperbolic tangent artifact model from [13]. Reductions in measured ICP pulsatility, e.g., when opening the external ventricular drain, are modeled by low-pass filtering the signal with a Butterworth filter with a cut-off frequency at $0.5 \mathrm{~Hz}$ (Fig. 10).

\section{Discussion and Conclusion}

We have presented an ICP simulator with dynamics on three time scales: Slow cerebrovascular and cerebrospinal dynamics, respiration-induced ICP variations and changes in ICP pulse amplitude and shape as a function of mean ICP. The synthetic ICP signals can be used to test a wide range of algorithms, from fiducial point extraction (peak

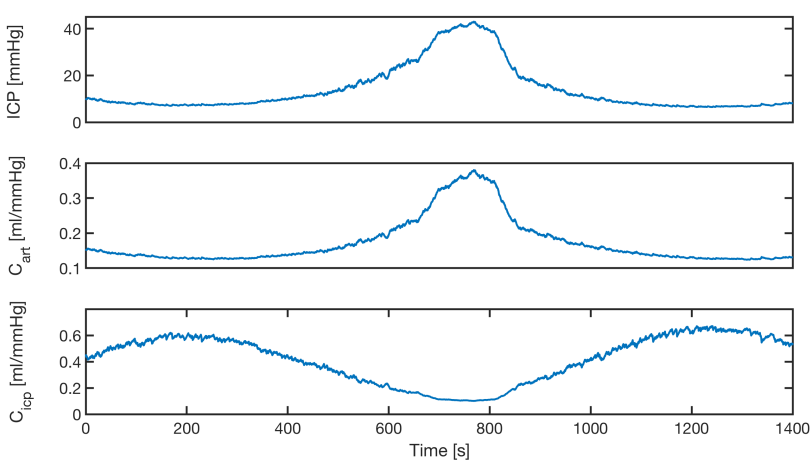

Figure 3: Simulated ICP plateau wave as well as arterial and intracranial compliance obtained by simulating the circuit model in Fig. 1.

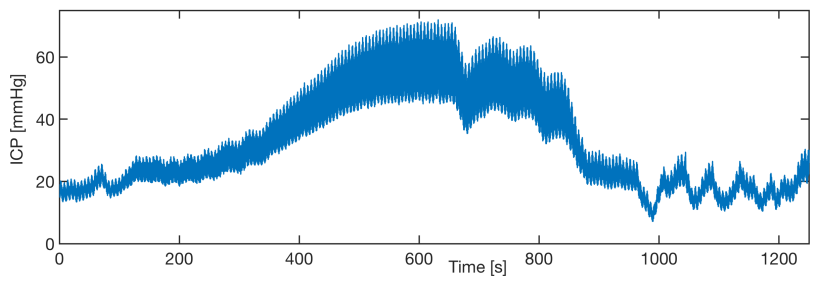

Figure 4: Real ICP recording with increased pulsatility during plateau wave (episode of intracranial hypertension).

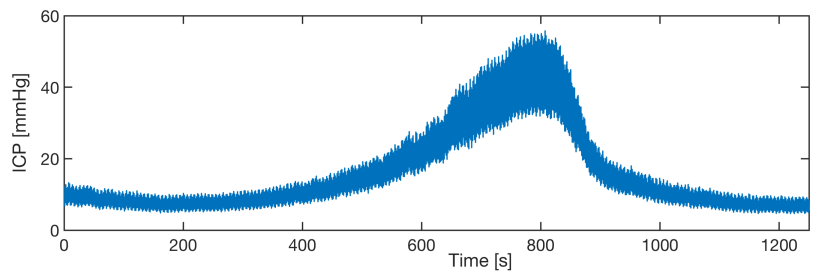

Figure 5: Simulated ICP plateau wave. The simulated signal shows less variability than the real signal in Fig. 4, since we do not model ICP Bwaves. These originate in vasogenic activity akin to ABP Mayer waves.

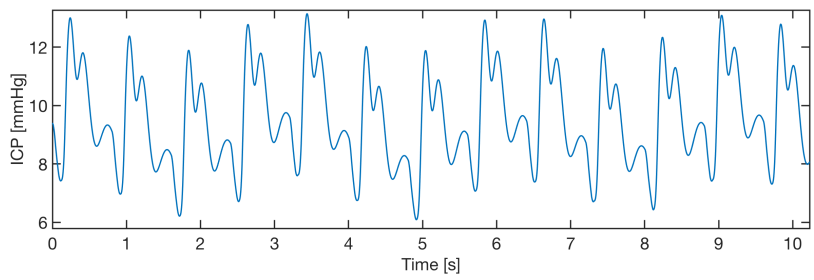

Figure 6: Simulated ICP signal with respiration-induced ICP amplitude variations. The peak P1 is the largest of the three, which is characteristic for ICP in a physiologic range.

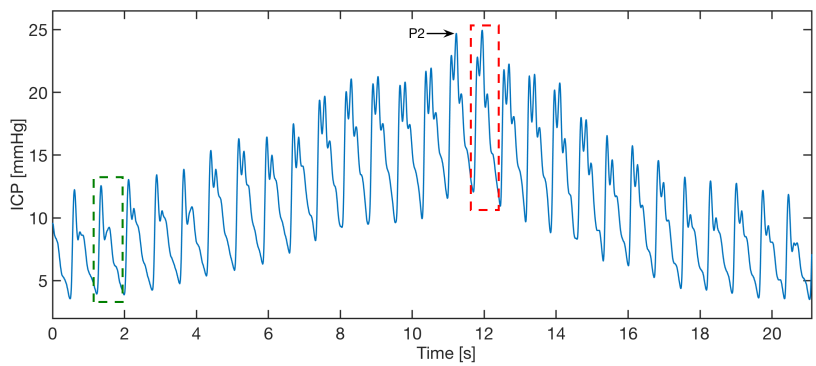

Figure 7: Real ICP recording with ICP pulse changing its shape as a function of mean ICP (P2 elevation at high ICP). 


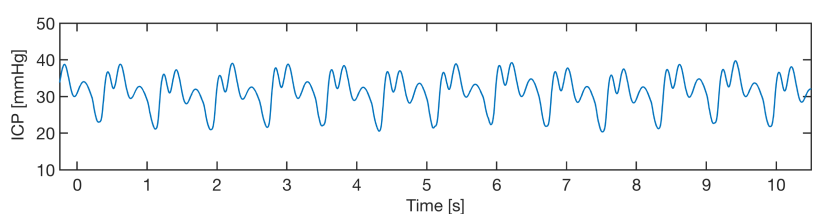

Figure 8: Simulated ICP signal with P2-elevations often present in elevated ICP and reduced intracranial compliance situations.

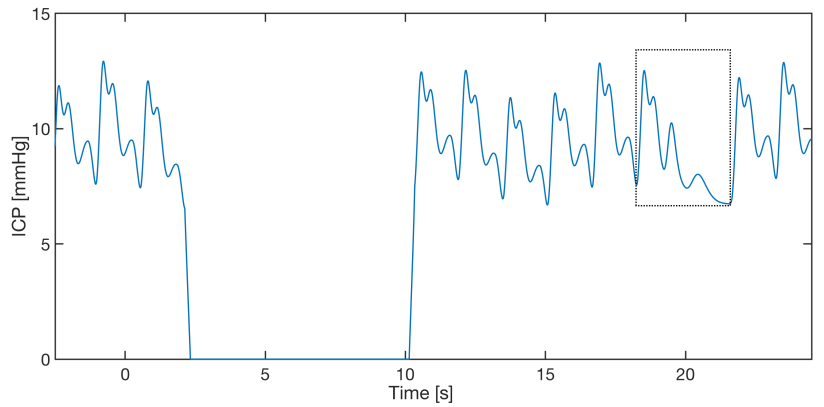

Figure 9: Simulated ICP signal with signal drop (e.g., due to brief unplugging of the sensor from the patient monitor) and beat followed by premature ventricular contraction (dotted box).

detection, onset detection, etc.) to signal quality assessment algorithms. A further potential use case is testing patient monitors, in particular ICP alarm settings. It is important to note that ICP is determined by multiple factors and that the neuroanatomy can vary significantly among patients. The described signal characteristics, therefore, are not necessarily representative of all patients. From a physiological perspective, the main short-coming of the simulator is that, unlike for slow ICP trends, both the respiration-induced ICP changes and the ICP pulses come from phenomenological models. These are aimed at generating realistically looking ICP signals, but lack an underlying mechanistic model describing causal relationships. Physiologically-based respiration and wave-propagation models describing the pulse shapes would be valuable for a causal understanding. For testing and benchmarking ICP analysis algorithms, however, capturing the relevant signal features is sufficient, as long as these reflect the real measurements. Finally, incorporating more control mechanisms into the simulator such as the Cushing triad (bradycardia, irregular breathing and widened pulse pressure) would further increase its level of realism.

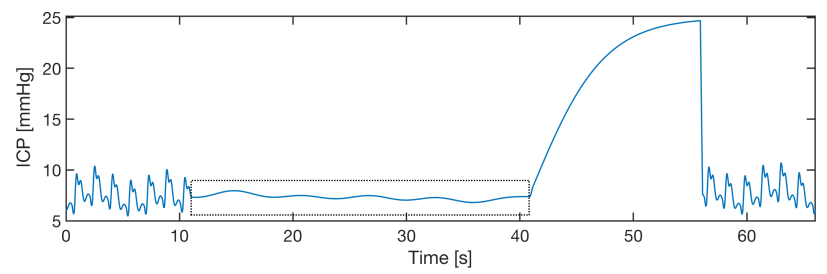

Figure 10: Simulated ICP signal with open external ventricular drain, leading to reduced pulsatility (dotted box), and catheter flush leading to an abnormally large and characteristically-shaped reading.

\section{References}

[1] Steiner L, Andrews P. Monitoring the injured brain: ICP and CBF. British Journal of Anaesthesia 2006;97:26-38.

[2] Balestreri M, Czosnyka M, Steiner L, Schmidt E, Smielewski P, Matta B, Pickard J. Intracranial hypertension: what additional information can be derived from ICP waveform after head injury? Acta Neurochirurgica 2004; 146(2):131-141.

[3] Kashif F, Verghese G, Novak V, Czosnyka M, Heldt T. Model-based noninvasive estimation of intracranial pressure from cerebral blood flow velocity and arterial pressure. Science Translational Medicine 2012;4(129):129-144.

[4] Wadehn F, Walser D, Bohdanowicz M, Czosnyka M, Heldt T. Non-invasive detection of intracranial hypertension using random forests. In Computing in Cardiology (CinC). Sept. $2017 ; 1-4$

[5] Hu X, Glenn T, Scalzo F, Bergsneider M, Sarkiss C, Martin $\mathrm{N}$, Vespa P. Intracranial pressure pulse morphological features improved detection of decreased cerebral blood flow. Physiological Measurement 2010;31(5):679.

[6] McSharry PE, Clifford GD, Tarassenko L, Smith LA. A dynamical model for generating synthetic electrocardiogram signals. IEEE Transactions on Biomedical Engineering 2003;50(3):289-294.

[7] Ursino M, Lodi CA. A simple mathematical model of the interaction between intracranial pressure and cerebral hemodynamics. Journal of Applied Physiology 1997; 82(4):1256-1269.

[8] Aboy M, McNames J, Hornero R, Thong T, Cuesta D, Novak D, Goldstein B. A novel statistical model for simulation of arterial and intracranial pressure. In 26th Annual International Conference of the IEEE Engineering in Medicine and Biology Society, volume 1. September 2004; 129-132.

[9] Wakeland W, Goldstein B. A review of physiological simulation models of intracranial pressure dynamics. Computers in Biology and Medicine 2008;38(9):1024-1041.

[10] Hall JE. Guyton and Hall Textbook of Medical Physiology. 13 edition. Elsevier Health Sciences, 2015.

[11] Carrera E, Kim DJ, Castellani G, Zweifel C, Czosnyka Z, Kasprowicz M, Smielewski P, Pickard JD, Czosnyka M. What shapes pulse amplitude of intracranial pressure? Journal of Neurotrauma 2010;27(2):317-324.

[12] Avezaat C, Van Eijndhoven J, Wyper D. Cerebrospinal fluid pulse pressure and intracranial volume-pressure relationships. Journal of Neurology Neurosurgery Psychiatry 1979;42(8):687-700.

[13] Li Q, Mark RG, Clifford GD. Artificial arterial blood pressure artifact models and an evaluation of a robust blood pressure and heart rate estimator. Biomedical Engineering Online 2009;8(1):13.

Address for correspondence:

Federico Wadehn,

wadehn@isi.ee.ethz.ch,

8092 Zurich, Switzerland. 